\title{
To Study the Effect of Swertiamarin in Animal Model of Huntington's Disease
}

\section{Rahul PG' ${ }^{1}$, Rahul WG ${ }^{2}$ and Sameer SS ${ }^{3}$.}

1Principal, Uttamrao Deshmukh Institute of Pharmacy, India

${ }^{2}$ Assistent Professor, Durgamata Institute of Pharmacy, India

${ }^{3}$ Principal, Durgamata Institute of Pharmacy, India

*Corresponding author: Sameer S Sheaikh, Principal, Durgamata Institute of Pharmacy, Dharmapuri, Parbhani, Maharashtra, India, Tel: +91 7972164935; Email: sameer_sheaikh80@rediffmail.com

\section{Research Article}

Volume 2 Issue 3

Received Date: September 11, 2018

Published Date: November 05, 2018

DOI: $10.23880 /$ oajpr-16000163

\section{Abstract}

Objective: The Data suggest that swertiamarin has antioxidant activity and they modulate neurotoxicity induced oxidative impairments in the brain and can be effectively employed as a neuroprotective. The preclinical findings obtained in the present study may provide a rationale for clinical trials of swertiamarin in humans with HD. Swertiamarin is a safe and well tolerated drug used as nutrient so can be administered for a long period of time.

Method: Adult male Wistar rats born and reared in the Animal House of the Agnihotri College of Pharmacy, Wardha, India was used in the present study. Young healthy male rats (250-300 g) were group housed (Six per cage) and maintained at $23 \pm 2^{\circ} \mathrm{C}$ under 12:12 hrs light (08:00-20:00 h)/dark cycle with free access to rodent chow and tap water. The animal studies were approved by the Institutional Animal Ethics Committee, constituted for the purpose of control and supervision of experimental animals by Ministry of Environment and Forests, Government of India, New Delhi, India. Animals were naive to drug treatments and experimentation at the beginning of all studies. All tests were conducted between 08:00 and13:00 h. The drug was tested in doses of 25-100 mg/kg ip. Unless stated otherwise, the data given indicate the effect of swertiamarin in doses of $50 \mathrm{mg} / \mathrm{kg}$ ip and a pretreatment time of $1 \mathrm{hr}$.

Result: These findings demonstrate that daily treatment with swertiamarin protects against various behavioral and biochemical alterations induced by 3-nitropropionic acid in rats. However, further studies are required to understand the exact mechanism involved in its neuroprotective role in this animal model of Huntington's disease.

Conclusion: Swertiamarin treatment protects behavioral changes, and significantly attenuated oxidative damage and improved mitochondrial complexes enzyme activities in different regions (striatum, cortex and hippocampus) of rat brain against 3-NP induced neurotoxicity. The results show that more effective than Swertiamarin and thus it could be used as an effective therapeutic agent in the management of Huntington's disease and related conditions. 


\section{Open Access Journal of Pharmaceutical Research}

Keywords: Huntington's disease; Huntingtin gene; 3-Nitropropionic Acid; Swertiamarin; Neurotoxicity

\section{Introduction}

Huntington's disease, or disorder (HD), is a neurodegenerative genetic disorder that affects muscle coordination and leads to cognitive decline and dementia. It typically becomes noticeable in middle age. HD is the most common genetic cause of abnormal involuntary writhing movements called chorea and is much more common in people of Western European descent than in those from Asia or Africa. The disease is caused by an autosomal dominant mutation on either of an individual's two copies of a gene called Huntingtin, which means any child of an affected parent has a $50 \%$ risk of inheriting the disease [1]. In rare situations where both parents have an affected copy this risk increases to $75 \%$, and when either parent has two affected copies, the risk is $100 \%$ (all children will be affected). Physical symptoms of Huntington's disease can begin at any age from infancy to old age, but usually begin between 35 and 44 years of age. About $6 \%$ of cases start before the age of 21 years with an akinetic-rigid syndrome; they progress faster and vary slightly. The variant is classified as juvenile, akinetic-rigid or Westphal variant HD [2].

\section{Signs and Symptoms}

The most characteristic initial physical symptoms are jerky, random, and uncontrollable movements called chorea. Chorea may be initially exhibited as general restlessness, small unintentionally initiated or uncompleted motions, lack of coordination, or slowed saccadic eye movements. These minor motor abnormalities usually precede more obvious signs of motor dysfunction by at least three years. The clear appearance of symptoms such as rigidity, writhing motions or abnormal posturing appear as the disorder progress [3] (Tables 1 and 2).

\begin{tabular}{|c|c|}
\hline Irritability & $38-73 \%$ \\
\hline Apathy & $34-76 \%$ \\
\hline Anxiety & $34-61 \%$ \\
\hline Depressed mood & $33-69 \%$ \\
\hline Obsessive and compulsive & $10-52 \%$ \\
\hline Psychotic & $3-11 \%$ \\
\hline
\end{tabular}

Table 1: Behavioral and psychiatric symptoms in Huntington's disease.

\begin{tabular}{|c|c|c|}
\hline Repeat count & Classification & Disease status \\
\hline$<28$ & Normal & Unaffected \\
\hline $28-35$ & Intermediate & Unaffected \\
\hline $36-40$ & $\begin{array}{c}\text { Reduced } \\
\text { Penetrance }\end{array}$ & $+/$ - Affected \\
\hline$>40$ & Full Penetrance & Affected \\
\hline
\end{tabular}

Table 2: Classification of the trinucleotide repeat, and resulting disease status, depends on the number of CAG repeats.

\section{Literature Review}

It is also characterized by degeneration of the striatal neurons, which leads to both motor and cognitive impairments. Previous reports suggest that excitotoxic events, oxidative stress, alterations in energy metabolism and mitochondrial dysfunction are involved in the pathophysiology of HD. The generation of ROS and the resulting oxidative damage are thought to play a central role in the process of neurodegeneration. Overproduction of reactive oxygen species (ROS) in neurons is physiologically blocked by free radical scavengers, such as glutathione and superoxide dismutase, in the body [4] (Figures 1 and 2).

\section{Drug Profile \\ Inducer Drug}

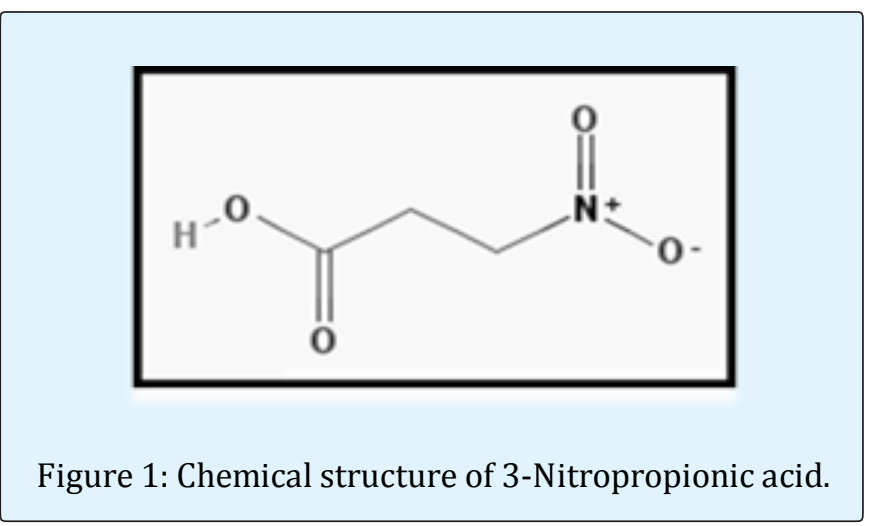




\section{Open Access Journal of Pharmaceutical Research}

\section{Treatment Drug}

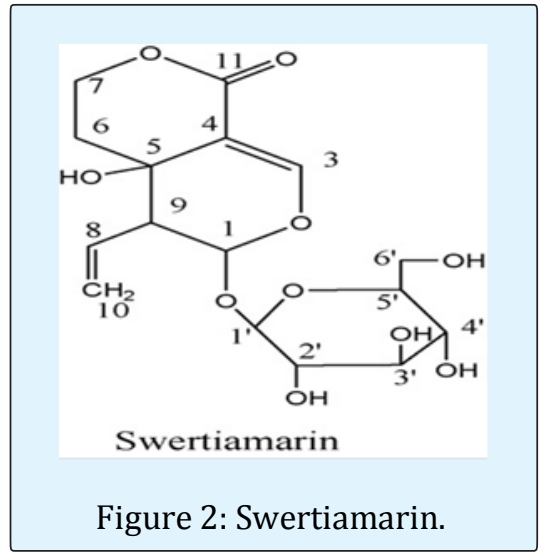

\section{Objective and Plan of Work}

Grouping and Randomization. Induction of Huntington's disease using 3-nitropropoinic acid. Fourteen day daily i.p. dosing of Swertiamarin and oral dosing of Cod liver oil $1 \mathrm{hr}$ before 3-nitropropoinic acid. To determine the effect Swertiamarin in animal model of Huntington's disease. Termination on day 14 and parameters to be measured as follows:

a. Succinate dehydrogenase

b. Lactate dehydrogenase

c. Reduced glutathione

d. Lipid peroxidation

e. Acetylcholinesterase

f. Nitrite

\section{Materials and Method}

\section{Subject}

Adult male Wistar rats born and reared in the Animal House of the Agnihotri College of Pharmacy, Wardha, India was used in the present study. Young healthy male rats (250-300 g) were group housed (Six per cage) and maintained at $23 \pm 2^{\circ} \mathrm{C}$ under $12: 12$ hrs light (08:00-20:00 h)/dark cycle with free access to rodent chow and tap water. The animal studies were approved by the Institutional Animal Ethics Committee, constituted for the purpose of control and supervision of experimental animals by Ministry of Environment and Forests, Government of India, New Delhi, India. Animals were naive to drug treatments and experimentation at the beginning of all studies. All tests were conducted between 08:00 and13:00 h.

\section{Drugs and Solutions}

The drug was tested in doses of $25-100 \mathrm{mg} / \mathrm{kg}$ ip. Unless stated otherwise, the data given indicate the effect of swertiamarin in doses of $50 \mathrm{mg} / \mathrm{kg}$ ip and a pretreatment time of $1 \mathrm{hr}$ [5].

\section{Treatments Schedule}

1. The following drugs were used in the present study. 3NP (Sigma Chemical, India) was diluted with saline (adjust pH 7.4) and administered intraperitoneally to animals. Swertiamarin sulphate (Sigma-Aldrich Labs, Bangalore, India) was diluted with double saline and administered intraperitoneally, DHA (Docosahexaenoic acid) administered by per oral route in a constant volume of $0.5 \mathrm{ml} / 100 \mathrm{~g}$ of body weight.

2. Animals were randomly divided into twelve groups of 6 animals in each. Group-1 vehicle treated, received normal saline (i.p.); Group-2 received 3-NP (10 $\mathrm{mg} / \mathrm{kg}$, i.p.) for14days Group-3 received Swertiamarin $(10 \mathrm{mg} / \mathrm{kg})$ per se, Group-4, 5, 6, received Swertiamarin $(2.5,5,10 \mathrm{mg} / \mathrm{kg})+3-\mathrm{NP}(10$ $\mathrm{mg} / \mathrm{kg}$, i.p.) for 14 days. Swertiamarin was administered $1 \mathrm{~h}$ prior to 3-NP administration [6].

3. Group-7 vehicle treated, received corn oil (p.o.); Group-8 received 3-NP (10 mg/kg, i.p.) for 14 days, Group-9 received DHA (100mg/kg) per se, Group$10,11,12$, received DHA $(50,100,200 \mathrm{mg} / \mathrm{kg})+3-\mathrm{NP}$ $(10 \mathrm{mg} / \mathrm{kg}$, i.p.) for 14 days. DHA was administered $1 \mathrm{~h}$ prior to 3-NP administration.

\section{Behavioral Tests}
a. Morris water maze test (Figures 4 and 5)
b. Novel object recognition test (Figure 7)
c. Actophotometer test (Figure 3)
d. Limb withdrawal test (Figure 6)

\section{Biochemical Estimations}

a. Estimation of Acetyl cholinesterase activity (Figure 10)

b. Estimation of lipid peroxidation (Figure 11)

c. Estimation of glutathione (Figure 12)

d. Estimation of nitrite (Figure 13)

e. Succinate dehydrogenase (SDH) activity (Complex-II)

(Figure 8)

f. Lactate dehydrogenase enzyme activity (Figure 9)

g. Protein estimation

\section{Results}

Effect of daily treatment of swertiamarin on 3nitropropionic acid-induced alterations in various behavioral parameters.

Effect of swertiamarin on locomotor activity: 


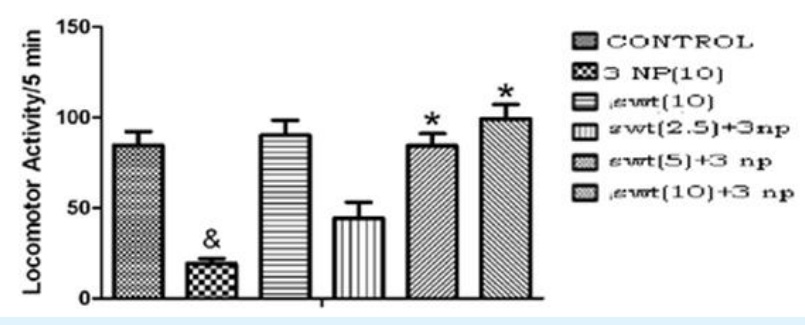

Figure 3: Influence of swertiamarin treatment on locomotor activity in Actophotometer.

Effect of swertiamarin on spatial navigation task in 3NP treated rats:

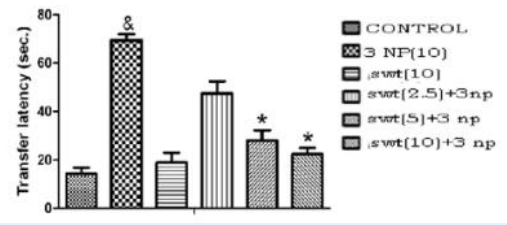

Figure 4: Influence of swertiamarin treatment on transfer latency in Morris water maze.

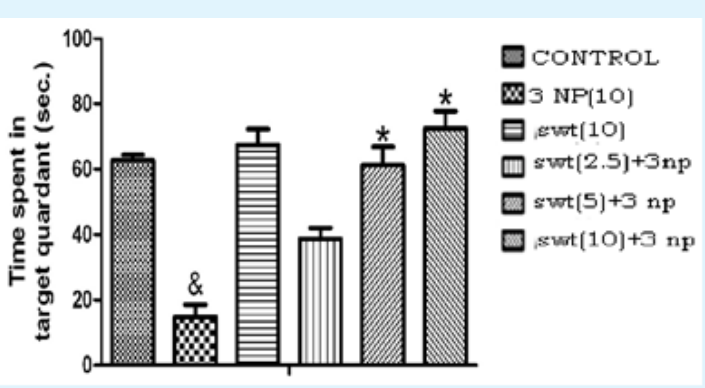

Figure 5: Influence of swertiamarin treatment on time spent in target quadrant in Morris water maze.

Effect of swertiamarin on limb withdrawal test:

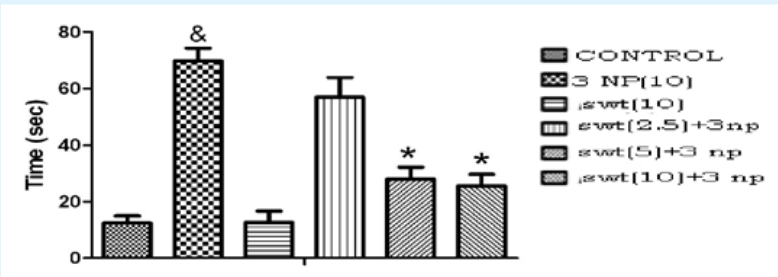

Figure 6: Influence of swertiamarin treatment on the limb withdrawal.
Effect of swertiamarin on Novel object recognition memory:

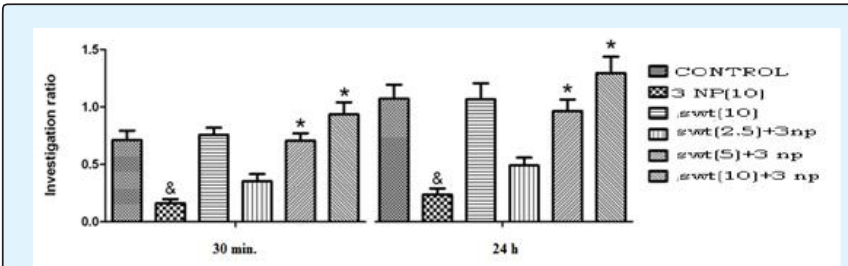

Figure 7: Influence of treatment of swertiamarin on the investigation ratios for the two retention intervals (30 $\mathrm{min}$ and $24 \mathrm{~h}$ respectively), for the object recognition test.

Effect of daily treatment of Swertiamarin on 3nitropropionic acid-induced alterations in various biochemical parameter

Effect of swertiamarin on succinate dehydrogenase enzyme:

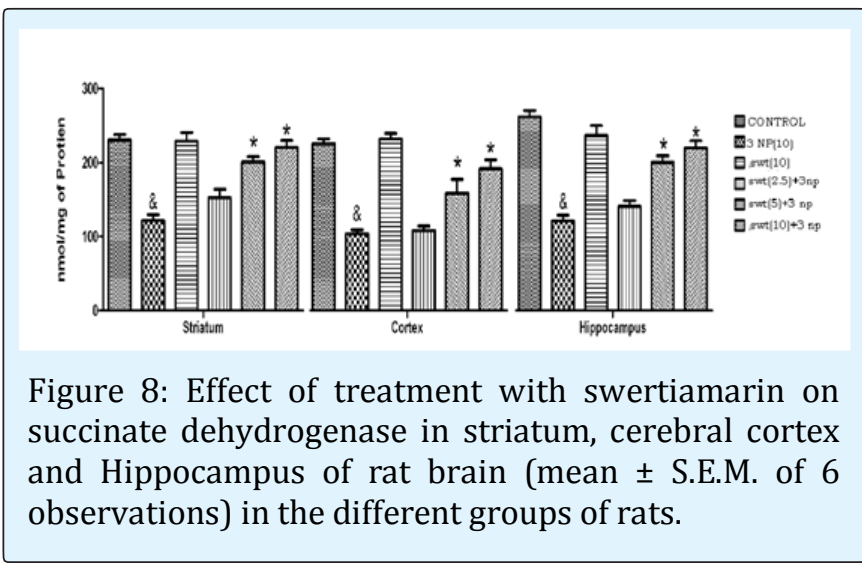

Effect of swertiamarin on the lactate dehydrogenase activity in 3-NP treated rats:

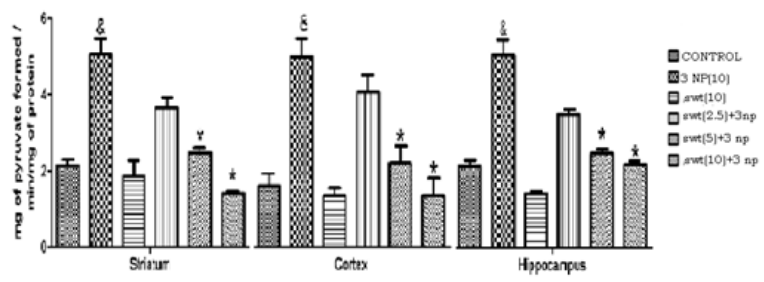

Figure 9: Effect of treatment with swertiamarin on lactate dehydrogenase in striatum, cerebral cortex and Hippocampus of rat brain (mean \pm S.E.M. of 6 observations) in the different groups of rats. 
Effect of swertiamarin on acetyl cholinesterase levels in 3-NP treated rats:

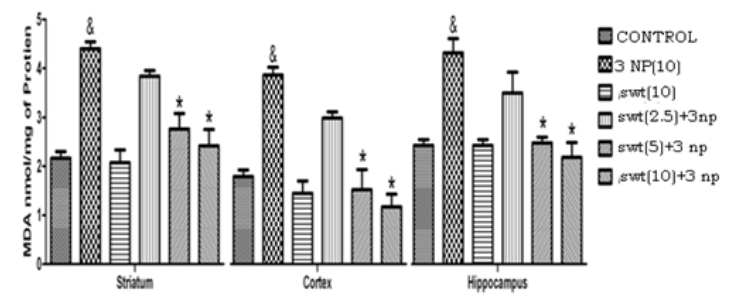

Figure 10: Effect of treatment with swertiamarin on acetyl cholinesterase level in striatum, cerebral cortex and hippocampus of rat brain (mean \pm S.E.M. of 6 observations) in the different groups of rats.

Effects of swertiamarin on parameters of oxidative stress in brain:

Effect of swertiamarin on lipid peroxidation (MDA)

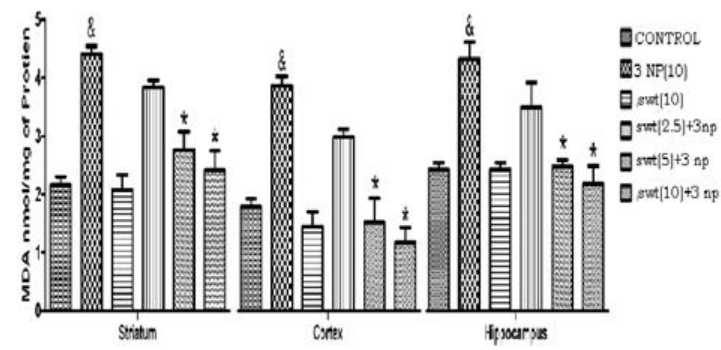

Figure 11: Effect of treatment with swertiamarin on lipid peroxidation in striatum, cerebral cortex and Hippocampus of rat brain (mean \pm S.E.M. of 6 observations) in the different groups of rats.

\section{Effect of swertiamarin on reduced glutathione:}

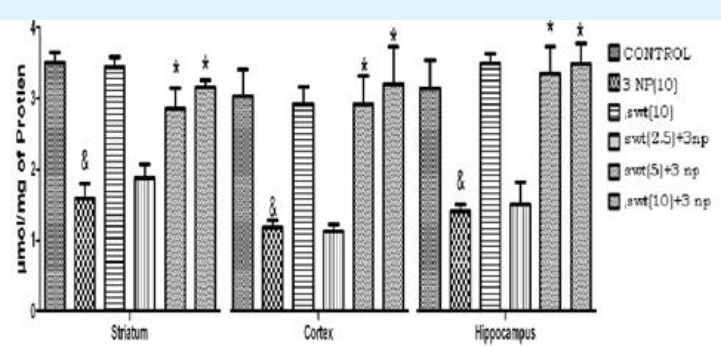

Figure 12: Influence of treatment with swertiamarin on glutathione levels in striatum, cerebral cortex and hippocampus of rat brain (mean \pm S.E.M. of 6 observations) in the different groups of rats.

\section{Effect of swertiamarin on nitrite concentration:}

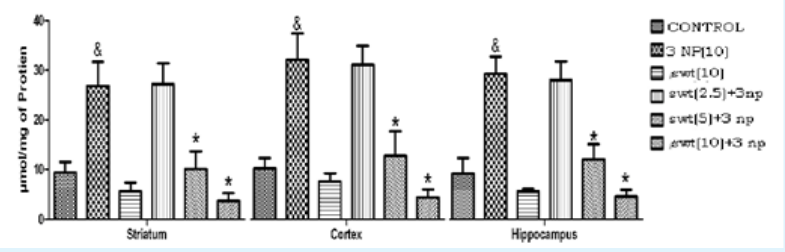

Figure 13: Influence of treatment with swertiamarin on nitrite concentration in striatum, cerebral cortex and hippocampus of rat brain (mean \pm S.E.M. of 6 observations) in the different groups of rats.

\section{Discussion}

The core finding of the present study is that administration of relevant doses of swertiamarin is remarkably neuroprotective in rats against 3nitropropionic acid-induced neurotoxicity. We have chosen the dose of swertiamarin $(2.5,5$ and $10 \mathrm{mg} / \mathrm{kg}$., i.p.) according to the previous studies done in our laboratory [7]. There are no previous reports on the protective effect of swertiamarin in 3-nitropropionic acid induced neurotoxicity, an animal model for Huntington's disease. In the present study, swertiamarin attenuated various behavioral and biochemical alterations due to 3nitropropionic acid and thus providing the first evidence regarding its beneficial effect in Huntington's disease [8].

Huntington's disease is a hereditary autosomal dominant neurodegenerative disease characterized by motor, cognitive and psychiatric symptoms. Huntington's disease patients show degenerative changes in the basal ganglia structures particularly caudate and putamen, resulting in a shrunkend brain and enlarged ventricles. Although the striatum is the most profoundly affected region in HD, degenerative changes in the cortex, thalamus, and subthalamic nucleus have also been reported in HD [9]. Though the exact mechanism of neuronal degeneration in HD is obscure, several reports have hypothesized the enhanced glutamate neurotransmission, apoptosis, transcriptional dysregulation, oxidative stress, and mitochondrial dysfunction and neurotransmitter alterations [10].

The present study employed 3-nitropropionic acid as an animal model of Huntington's disease (HD). 3Nitropropionic acid (3-NP) is a suicide inhibitor of succinate dehydrogenase and is a widely distributed plant and fungal neurotoxin known to induce damage to basal ganglia, hippocampus, spinal tracts and peripheral nerves 


\section{Open Access Journal of Pharmaceutical Research}

in animals. Reduced activity of the complex II enzyme succinate dehydrogenase ( $\mathrm{SDH}$ ) has been found in postmortem HD brain tissue [11]. In addition, a decrease in complex II activity in HD basal ganglia has been observed. A major advantage of this model over other models of HD is that the lesions produced are bilateral and striatalspecific, and develops spontaneously after systemic administration of 3-NP [12].

Systemic administration of 3-nitropropionic acid is known to produce hypoactivity that resembles juvenile onset and advanced Huntington's disease in rats [13]. It produces significant motor and behavioral abnormalities including bradykinesia, muscles weaknesses and rigidity [14]. These findings are in agreement with earlier reports which also observed a variety of neurobehavioral abnormalities and motor deficit in rats following 3-NP administration $[15,16]$. In the present study, administration of 3-nitropropionic acid daily for 14 days decreased the ambulatory movements (in actophotometer) and causes a delay in retraction time of the hind limb (in Limb withdrawal apparatus), thus representing the motor abnormalities [17]. Daily treatment with swertiamarin for 14 days dosedependently attenuated 3-nitropropionic acid-induced hypolocomotion and motor incoordination [1].

Systemic administration of 3-nitropropionic acid also decreased the SDH levels in the whole brain, suggesting mitochondrial damage and pre-treatment with swertiamarin attenuated this decrease in SDH levels. These results show that swertiamarin may prevent mitochondrial deterioration and maintain synaptic integrity against damage induced by 3-nitropropionic acid [11].

3-NP is known to produce memory deficit which could be related to its selective striatal damage and certain hippocampal neuronal damage [17]. Among the affected neurons in the hippocampal complex are the pyramidal neurons in the CA1 region as well as various neurons in the CA3 region and dentate hilar area [18]. Memory loss may be due to an increase in the brain oxidative stress, which seems to have an important role in cognitive impairment caused by normal aging and neurodegenerative diseases [7].

In the present study, 3-nitropropionic acid produced memory deficit in rats and daily treatment with swertiamarin improved memory retention in 3-NP. This property of swertiamarin could be helpful in ameliorating the memory impairment in Huntington's disease patients.
Increased oxidative stress and mitochondrial abnormalities contribute to neuronal dysfunction in HD. The mitochondrial genome may play an essential role in the pathogenesis of various neurodegenerative diseases including HD, and evidence for mitochondria being a site of damage in neurodegenerative disorders is based in part on observed decreases in the respiratory chain complex activities in Parkinson's, Alzheimer's, and Huntington's disease. Such defects in respiratory complex activities, possibly associated with oxidant/antioxidant imbalance, are thought to underlie defects in energy metabolism and induce cellular degeneration [7]. Free radicals typically generated from mitochondrial respiration, cause oxidative damage of nucleic acids, lipids, carbohydrates and proteins.

It was suggested that plasma malondialdehyde may be used as a potential biomarker to test treatment efficacy of drugs used in HD [9]. A significant amount of work has suggested that free radical generation may underlie the neurotoxic effects of succinate dehydrogenase inhibitors such as 3-NP. In the present study, there was an increase in the brain MDA (indicator of the lipid peroxidation due to free radicals) and decrease in reduced glutathione levels following 3-NP administration, which was attenuated by swertiamarin, suggesting the antioxidant action of swertiamarin. Moreover, there was increase in nitrite levels with 3-nitropropionic acid which was reversed by swertiamarin [16].

Cholinergic neurotransmission is a central process underlying memory and cognitive function. Cholinergic basal forebrain neurons in the nucleus basalis magnocellularis innervate the cerebral cortex, amygdaloid complex and hippocampus, and are essential for learning and memory formation [5]. One of the most important mechanisms responsible for correct cholinergic function is performed by enzyme choline esterase (ChE). In the present study, treatment with swertiamarin partially decreased the levels of ChE in cerebral cortex and hippocampus of HD rats [19].

These findings demonstrate that daily treatment with swertiamarin protects against various behavioral and biochemical alterations induced by 3-nitropropionic acid in rats. However, further studies are required to understand the exact mechanism involved in its neuroprotective role in this animal model of Huntington's disease [20].

In the present study, 3-NP treatment produced significant motor and behavioral abnormalities including bradykinesia, muscle weakness and rigidity. These finding 


\section{Open Access Journal of Pharmaceutical Research}

are in agreement with earlier reports which also observed a variety of neurobehavioral abnormalities and motor deficit in rats following 3-NP administration. The symptoms developed by chronic administration of 3-NP are analogues to juvenile onset and late hypokinetic stages of HD. 3-NP administration for 14 days significantly impaired body weight and locomotor activity of the animals as compare to the vehicle treated group. The striatal lesions and bradykinesia could be partly responsible for reducing rat appetite, food intake and motor incoordination [8]. Treatment with the DHA significantly reversed the alteration in body weight and locomotor activity. Motor activity of the 3-NP treated animals also significantly decreased in rot actophotometer as compared to the vehicle treated rats and impaired motor changes were significantly reversed by DHA treatment [12].

Systemic administration of the mitochondrial toxin, 3NP leads to depletion of cellular ATP and induces striatum-specific lesions that resemble Huntington's like disease. 3-NP treatment significantly depleted enzyme levels (succinate dehydrogenase and reduced glutathione) and raised oxidants (increased lipid peroxidation, nitrite level) suggesting the role of oxidative stress in the neurodegenerative process [21].

Previous studies clearly demonstrate that increased oxidative stress can be one of the major deleterious events in clinical and experimentally induced HD. In the present study, 3-NP significantly increased oxidative stress (lipid peroxidation and nitrite levels) and decreased enzyme levels (succinate dehydrogenase and reduced glutathione) in striatum, cortex and hippocampal regions of the brain, which were reversed by DHA treatment [18].

Furthermore, neurotoxicity of 3-NP is attributed to its ability to produce ischemic injury by interfering with complex-II of mitochondrial respiratory chain leading to depressed ATP levels. In this study, 3-NP significantly decreased mitochondrial complex activity (Complexes- I, II, III, and IV) in the striatum, cortex and hippocampal areas of brain. Complex-II enzyme activity was significantly reduced as compared to other complexes because of the specificity of 3-NP towards succinate dehydrogenase [7]. Neurons in brain are highly vulnerable to ischemia and therefore impairment of energy metabolism was associated with neurodegenerative changes including HD in brain. DHA treatment for 14 days significantly attenuates the dysfunctional mitochondrial enzyme complex in striatum, cortex and hippocampus [10,22,23].

Sameer SS, et al. To Study the Effect of Swertiamarin in Animal Model of Huntington's Disease. Pharm Res 2018, 2(3): 000163.

\section{Conclusions and Future Scope}

Swertiamarin treatment protects behavioral changes, and significantly attenuated oxidative damage and improved mitochondrial complexes enzyme activities in different regions (striatum, cortex and hippocampus) of rat brain against 3-NP induced neurotoxicity. The results show that more effective than Swertiamarin and thus it could be used as an effective therapeutic agent in the management of Huntington's disease and related conditions.

We attempted to investigate the neuroprotective effect of Swertiamarin in animal model of Huntington's disease. To get a detailed account of the Swertiamarin in neuroprotection further confirmatory studies are required. Immunocytochemical studies should be carried out to further strengthen the neuroprotective effect of Swertiamarin. To study the effect of Swertiamarin in transgenic animal model of HD.

\section{References}

1. Beckman JS (1996) Oxidative damage and tyrosine nitration from peroxynitrite. Chem Res Toxicol 9(5): 836-844.

2. Borlongan CV, Kanning $\mathrm{K}$, Poulos SG, Freeman TB, Cahill DW, et al. (1996) Free radical damage and oxidative stress in Huntington's disease. J Fla Med Assoc 83(5): 335-341.

3. Blantz RC, Satriano J, Gabbai F, Kelly C (2000) Biological effects of arginine metabolites. Acta Physiol Scand 168(1): 21-25.

4. Brouillet E, Conde F, Beal MF, Hantraye P (1999) Replicating Huntington's disease phenotype in experimental animals. Prog Neurobiol 59(5): 427-468.

5. Borlongan CV, Koutouzis TK, Freeman TB, CahillDW, Sanberg PR (1995) Behavioral pathology induced by repeated systemic injections of 3-nitropropionic acid mimics the motoric symptoms of Huntington's disease. Brain Res 697(1-2): 254-257.

6. Kalmijn S, Launer LJ, Ott A, Witteman JC, Hofman A, et al. (1997) Dietary fat intake and the risk of incident dementia in the Rotterdam Study. Ann Neurol 42(5): 776-782.

7. Yamahara J, Kobayashi M, Matsuda H, Aoki S (1990-1) Anticholinergic action of Swertia juportku and an active constituent. Journal of Ethnopharmaeology 33(1-2): 31-35.

8. Hoth KF, Paulsen JS, Moser DJ, Tranel D, Clark LA, et al. 


\section{Open Access Journal of Pharmaceutical Research}

(2007) Patients with Huntington's disease have impaired awareness of cognitive, emotional, and functional abilities. J Clin Exp Neuropsychol 29(4): 365-376.

9. Huang LS, Sun G, Cobessi D, Wang AC, Shen JT, et al. (2006) 3-Nitropropionic acid is a suicide inhibitor of mitochondrial respiration that, upon oxidation by complex II, forms a covalent adduct with a catalytic base arginine in the active site of the enzyme. J Biol Chem 281(9): 5965-5972.

10. Cooper DB, Ales G, Lange C, Clement P (2006) Atypical onset of symptoms in Huntington disease: severe cognitive decline preceding chorea or other motor manifestations. Cogn Behav Neurol 19(4): 222224.

11. Duan W, Peng Q, Masuda N, Ford E, Tryggestad E, et al. (2008) Sertraline slows disease progression and increases neurogenesis in N171-82Q mouse model of Huntington's disease. Neurobiol Dis 30(3): 312-322.

12. Deshpande SB, Hida H, Takei-Io N, Masuda T, Baba H, et al. (2006) Involvement of nitric oxide in 3nitropropionic acid-induced striatal toxicity in rats. Brain Res 1108(1): 205-215.

13. Cipriani S, Bizzoco E, Gianfriddo $M$, Melani A, Vannucchi MG, et al. (2008) Adenosine A2A receptor antagonism increases nNOS-immunoreactive neurons in the striatum of Huntington transgenic mice. Exp Neurol 213(1): 163-170.

14. Duan W, Guo Z, Mattson MP (2000) Participation of par-4 in the degeneration of striatal neurons induced by metabolic compromise with 3-nitropropionic acid. Exp Neurol 165(1): 1-11.

15. Borlongan CV, Koutouzis TK, Sanberg PR (1997) 3Nitropropionic acid animal model and Huntington's disease. Neurosci Biobehav Rev 21(3): 289-293.

16. Gil JM, Rego AC (2008) Mechanisms of neurodegeneration in Huntington's disease. Eur J Neurosci 27(11): 2803-2820.

17. Guyot MC, Palfi S, Stutzmann JM, Mazière M, Hantraye P, et al. (1997) Riluzole protects from motor deficits and striatal degeneration produced by systemic 3nitropropionic acid intoxication in rats. Neuroscience 81(1): 141-149.

18. Karanian DA, Baude AS, Brown QB, Parsons CG, Bahr BA (2006) 3-Nitropropionic acid toxicity in hippocampus: protection through N-methyl-Daspartate receptor antagonism. Hippocampus 16(10): 834-842.

19. Borlongan CV, Koutouzis TK, Randall TS, Freeman TB, Cahill DW, et al. (1995b) Systemic 3-nitropropionic acid: behavioral deficits and striatal damage in adult rats. Brain Res Bull 36(6): 549-556.

20. Chen CM, Wu YR, Cheng ML, Liu JL, Lee YM, et al. (2007) Increased oxidative damage and mitochondrial abnormalities in the peripheral blood of Huntington's disease patients. Biochem Biophys Res Commun 359(2): 335-340.

21. Alexi T, Hughes PE, Faull RL, Williams CE (1998) 3Nitropropionic acid's lethal triplet: cooperative pathways of neurodegeneration. Neuroreport 9(11): R57-64.

22. Appleton KM, Hayward RC, Gunnel D, Peters TJ, Rogers PJ, et al. (2006) Effects of n-3 long-chain polyunsaturated fatty acids on depressed mood: systematic review of published trials. Am J Clin Nutr 84(6): 1308-1316.

23. Araki W, Wurtman RJ (1997) Control of membrane phosphatidylcholine biosynthesis by diacylglycerol levels in neuronal cells undergoing neurite outgrowth. Proc Natl Acad Sci USA 94(22): 11946-11950.

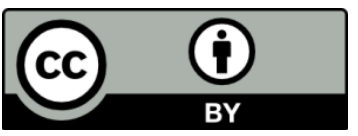

Sameer SS, et al. To Study the Effect of Swertiamarin in Animal Model of Huntington's Disease. Pharm Res 2018, 2(3): 000163. 